\title{
Linezolid Versus Vancomycin for the Treatment of Methicillin-Resistant Staphylococcus Aureus Acute Hematogenous Osteomyelitis in Children: Higher Anemia Rate During Therapy.
}

Chao Li

The Fourth Military Medical University

huifa xu

The Fourth Military Medical University

Zhichen Liu

The Fourth Military Medical University

yabo yan

The Fourth Military Medical University

\section{Weilong Diwu}

The Fourth Military Medical University

Jia Sha

The Fourth Military Medical University

Ge Li

Shaanxi Provincial Institute for Endemic Disease Control

\section{Zhexuan Guan}

The Fourth Military Medical University

\section{Luyu Huang ( $\square$ huangly@fmmu.edu.cn )}

The Fourth Military Medical University

\section{Research Article}

Keywords: Acute hematogenous osteomyelitis, Methicillin-resistant Staphylococcus aureus, Linezolid, Vancomycin, Anemia.

Posted Date: October 11th, 2021

DOl: https://doi.org/10.21203/rs.3.rs-877827/v1

License: (c) (i) This work is licensed under a Creative Commons Attribution 4.0 International License. Read Full License 


\section{Abstract}

Background: Clinical use of linezolid for the treatment of methicillin-resistant staphylococcus aureus (MRSA) acute hematogenous osteomyelitis (AHO) is still limited by lacking data about clinical outcomes and adverse effects of long-term usage in children. This study aimed to compare the efficacy and safety of linezolid and vancomycin for the treatment of MRSA AHO in children.

Methods: This retrospective study was conducted between January 2011 and December 2018 at the Xijing Hospital, Air Force Military Medical University, China, and compared the clinical efficacy and safety of linezolid and vancomycin in children with MRSA AHO. Demographics, clinical features, laboratory tests, susceptibilities of isolates, treatment outcomes, and adverse events were collected. Variables were analyzed by Fisher's exact test or Mann-Whitney U-test.

Results: 17 patients with MRSA AHO were included in this study ( 6 children received linezolid and 11 children received vancomycin). Statistically significant differenc was observed in the minimum hemoglobin during the treatment between 2 groups [71.0 (IQR 65.0-91.0) vs 102.0 (IQR 91.0-114.0) g/L, $P=0.009]$, and patients in the linezolid group presented higher anemia rate [5 (83.3\%) vs $3(27.3 \%)$, $\mathrm{P}=0.049]$ than those in the vancomycin group.

Conclusions: Although linezolid and vancomycin have no significant difference in efficacy and safety in the treatment of children with MRSA AHO, the incidence rate of anemia was higher during therapy. Children seemed to be more sensitive to the transient bone marrow suppression effect of linezolid in the prolonged use for MRSA AHO.

\section{Introduction}

Methicillin-resistant Staphylococcus aureus (MRSA) has become the predominant cause of acute hematogenous osteomyelitis (AHO) [1], which can lead to severe consequences due to producing enterotoxin B, phenol-soluble modulins, and Panton-Valentine leukocidin[2, 3].

Vancomycin is the first-line treatment and demonstrates positive clinical outcomes[4, 5]. However, its use has been related to some defects, such as potential nephrotoxicity and slow bactericidal activity[6].

Linezolid, as the first member of the oxazolidinone class approved for clinical use, has a broad spectrum of activity against nearly all Gram-positive pathogens including MRSA. Although it was recommended by the MRSA Infectious Diseases Society of America (IDSA) guidelines for pediatric AHO[7], its clinical use is still limited by sparse MRSA AHO data about clinical outcomes and adverse effects of long-term usage in children[8-11].

Given the increasing incidence of MRSA AHO and the lack of data comparing linezolid's clinical outcomes with other treatments on this disease, the aim of this study was to analyze the effectiveness and safety of linezolid and vancomycin for the treatment of MRSA AHO in children. 


\section{Materials And Methods}

\subsection{Study design}

We retrospectively reviewed all medical records of children ( $<18$ years old) who were carried a discharge diagnosis of AHO caused by MRSA and treated with at least one dose of linezolid or vancomycin from Xijing Hospital, Air Force Military Medical University (Xi'an, China) between January 2011 and December 2018. This retrospective study was approved by the Ethics Committee of Xijing Hospital. Two cohorts were defined, the MRSA AHO children patients admitted treated with either linezolid or vancomycin were labeled linezolid group and vancomycin group, respectively. All methods were carried out in accordance with relevant guidelines and regulations.

\subsection{Treatment}

Children in Linezolid group received doses of $10 \mathrm{mg} / \mathrm{kg} /$ dose every 8 hours in children $\leq 11$ years old, and $600 \mathrm{mg} / \mathrm{d}$ q12h in children $\geq 12$ years old, which were recommended and likely to be sufficient to reach pharmacodynamics targets for MRSA strains $[7,12]$. For linezolid, both oral and intravenous (IV) therapy were considered appropriate. Children in vancomycin group received vancomycin $15 \mathrm{mg} / \mathrm{kg} / \mathrm{dose}$ IV every 6 hours, which was in accordance with the MRSA IDSA treatment guidelines[7].

\subsection{Patients and variables}

All children patients who were enrolled in this study were identified by reviewing all medical records. Patients with acute osteomyelitis occurring after open wound, bone fracture, or surgery and patients with mixed culture results were excluded. Informed consent was obtained, as participants were under 18 , from parent and/or legal guardian. If the patient was hospitalized multiple times, we only analyzed his first medical record. After discharge, we followed patients for at least six months.

All data, which includes epidemiological, microbiological and clinical data, were collected by using predesigned forms. Age, sex, and race were included in epidemiological data. Duration of symptoms and previous therapy before admission, admission temperature, bone involvement, adjacent arthritis, susceptibilities of MRSA isolates and laboratory findings were included in clinical data. Treatment and outcome analyses included duration of antibiotic therapy, surgical procedures, duration of fever, clinical cure at the end of treatment (EOT) and at 28 days, and outcome.

We also recorded length of stay (LOS) and treatment-related adverse events. Special attention was paid to the manifestations of nausea, vomiting, diarrhea, skin rash, headache, peripheral neuropathy, red man syndrome (RMS), and visual impairment. The laboratory data of each case at the beginning of and throughout the duration of linezolid or vancomycin treatment, including routine hematology and serum chemistry assays and hepatorenal function test, were also collected to evaluate the extent of bone marrow suppression and hepatorenal impairment. We also analyzed inflammatory markers, such as Leukocyte, erythrocyte sedimentation rate (ESR) and C-reactive protein (CRP). 
We used microbiological tests to identify MRSA, including testing isolated MRSA for antibiotic sensitivity (oxacillin, linezolid, vancomycin, and clindamycin). Minimal inhibitory concentrations (MICs) of antibiotic were performed in sterile, flat-bottomed 96-well microplate according to the broth microdilution guidelines of Clinical and Laboratory Standards Institute (CLSI).[13]

\subsection{Definitions}

The diagnosis of MRSA AHO was based on the following criteria: presence of localized pain or tenderness and other typical features of osteomyelitis of no more than 15 days' duration, typical imaging manifestations, and bacteriologic evidence of MRSA infection (positive blood, tissue, or pyogenic fluid culture). Adjacent septic arthritis was considered when MRSA was isolated from synovial fluid from adjacent joint.

The definition of clinical cure was that no clinical and laboratory evidence of infection was detected after treatment of linezolid or vancomycin was discontinued. The definition of treatment failure was that treatment was stopped or changed before clinical cure can be achieved, which was usually due to persistent or progressive clinical or laboratory evidence of infection, or severe adverse events. An adverse reaction was defined as an adverse event that occurred after the use of linezolid or vancomycin and was suspected or proven to be associated with the use of the drug $[1,7,10,11,14-16]$. Relapse was defined as the isolation of a positive culture after clinical cure within 2 months. The criteria for the presence of druginduced liver injury (DILI) include any of the following $[17,18]$ : $\geq 5$-fold elevation above the upper limit of normal (ULN) for alanine aminotransferase (ALT) , $\geq 2$-fold elevation above the ULN for alkaline phosphatase (ALP), and $\geq 3$-fold elevation in ALT with simultaneous elevation of bilirubin exceeding two times the ULN. Normal baseline serum creatinine (SCr) value was defined as $\leq 53 \mu \mathrm{mol} / \mathrm{L}$ for patients $\leq$ $1 \mathrm{month}$ old and $\leq 72 \mu \mathrm{mol} / \mathrm{L}$ for those $>1$ month old. Nephrotoxicity was defined as SCr increase of $\geq 44 \mu \mathrm{mol} / \mathrm{L}$ or $\mathrm{a} \geq 50 \%$ increase in baseline SCr on at least two consecutive days[19]. RMS was defined as a hypersensitivity reaction by intravenous administration of vancomycin, with symptoms ranging from flushing, erythematous rash, pruritus, mild to profound hypotension, and even cardiac arrest[20, 21].Neutropenia was defined as an absolute neutrophil count of $<1.5 \times 10^{9} / \mathrm{L}$.

Thrombocytopenia was defined as a platelet count of $<150 \times 10^{9} / \mathrm{L}$. Anemia was defined as a hemoglobin of $<90 \mathrm{~g} / \mathrm{L}$.

\subsection{Statistical analysis}

Statistical analyses were performed using SPSS Statistics 23.0 (IBM Corp., Armonk, NY, USA). Percentage was used to present categorical variables, and Fisher's exact test was used to test whether there was statistical difference. Median [interquartile range (IQR)] was used to present continuous variables, and Mann-Whitney U-test was used to test the difference.

\section{Results}


A total of 49 children with discharge diagnosis of osteomyelitis and with MRSA-positive culture were identified during the 8-year study period. 29 patients whose time between symptom onset and admission was longer than 15 days were excluded. 3 cases of acute osteomyelitis (bone fracture, 2, plastic surgery, 1) were excluded. 17 cases of MRSA AHO that met inclusion criteria were analyzed as they received either linezolid or vancomycin. 6 patients received linezolid and 11 received vancomycin as definitive therapy for MRSA based on the results of sensitivity test and criteria of responsible professors.

Baseline epidemiological and clinical characteristics between treatment groups are shown in Table 1.

\subsection{Demographics}

Children with linezolid treatment had a median age of 8.5 (IQR 7.2-10.2) years, whereas those with vancomycin treatment had a median age of $13($ IQR 6.0-14.0) years $(P=0.131)$. There were 10 male and 7 female patients (male to female ratio: 1.4:1) with no significant gender differences observed between the 2 groups. The proportion of Han Chinese children in linezolid group did not differ significantly from that in vancomycin group.

\subsection{Clinical Features}

The duration between the onset of symptoms and admission in linezolid groups were 11.0 (IQR 6.5-14.5) days, and $4(66.7 \%)$ children received antibiotics before admission. these findings were similar to those from patients in vancomycin group. A recent illness presenting within 2 weeks before admission as an upper respiratory tract infection was reported in $1(16.7 \%)$ of the 6 patients with linezolid therapy. Two (33.3\%) children in linezolid group had history of preceding minor trauma. these findings were not significantly different from those of patients with vancomycin therapy. The admission temperature did not differ between treatment groups [37.5 (IQR 37.4-38.2) vs 37.8 (IQR 37.5-38.1) ${ }^{\circ} \mathrm{C}, \mathrm{P}=1.000$ ]. The most common sites of infection in MRSA AHO were femur (52.9\%) and tibia (29.4\%). One child had involvement of $>1$ bone. The frequency of occurrence of infection of different bones was similar between 2 groups. Concurrent adjoining septic arthritis was present in $33.3 \%$ and $27.3 \%$ of children treated with linezolid and vancomycin $(P=1.000)$, respectively.

\subsection{Laboratory findings}

Laboratory findings and inflammatory markers between treatment groups are shown in Table 2.

On admission, the median hemoglobin was 113.0 (IQR 97.3-124.7) and 118.0 (IQR 104.0-142.0) g/L for patients with linezolid and vancomycin, $(P=1.000)$, respectively. However, statistically significant difference was observed in minimum hemoglobin during the treatment between 2 groups [71.0 (IQR 65.091.0) vs 102.0 (IQR 91.0-114.0) $\mathrm{g} / \mathrm{L}, \mathrm{P}=0.009$ ]. While at the end of treatment, hemoglobin did not differ between 2 groups [107.0 (IQR 100.0-112.7) vs 106.0 (IQR 95.0-109.0). No statistically significant differences were observed in the count of platelet, neutrophil, and leukocyte, apart from higher neutrophil count on admission in the linezolid group $(P=0.049)$. No statistically significant differences were observed in ALT on admission [32.0 (IQR 12.7-62.7) vs 21.0 (IQR 7.0-42.0), P=1.000], during the treatment 
[15.5 (IQR 10.5-34.2) vs 19.0 (IQR 17.0-23.0), P=1.000], or at the EOT [12.0 (IQR 7.7-22.5) vs 22.0 (17.027.0), $P=0.304]$. At the EOT, the peripheral creatinine for patients in vancomycin group was significantly higher than that in linezolid group [64.0 (IQR 52.0-68.0) vs 37.0 (IQR 30.0-48.0), P=0.009]. However, significant difference was also observed in baseline creatinine between groups [61.0 (IQR 53.0-71.0) vs 40.5(IQR 33.2-51.2), $\mathrm{P}=0.009$ ]. The ESR and CRP did not differ between treatment groups on admission or at the EOT.

\subsection{Treatment and outcomes}

Both groups showed similar clinical and microbiological efficacy, as shown in Table 3.

There was no significant difference between the linezolid group and the vancomycin group in surgical frequency [2.0 (IQR 0.75-3.25) vs 2.0 (IQR 0.0-3.0), P=1.000]. In patients treated with linezolid, the median duration of antibiotic therapy, the median duration of fever after admission, and the length of hospitalization were 32.0 (IQR 25.7-57), 3.5 (IQR 2.0-7.0), and 30.0 (IQR 23.2-52.0) days, respectively. These findings were similar to those of patients treated with vancomycin. There were no differences concerning transfusion [2 (33.3\%) vs 1 (9.1\%), P=0.515], cure at EOT [6 (100\%) vs 9 (81.8\%), P=0.515], cure at 28 days [3 (50.0\%) vs $4(36.4 \%), P=0.644]$, and relapse [1 (16.7\%) vs $2(18.2 \%), P=1.000$ ] in the linezolid and vancomycin groups.

Antibacterial susceptibilities for 17 isolated of MRSA are shown in Table 4.

\subsection{Adverse events}

The rate of adverse events compared by treatment is shown in Table 5. Although not significant, the treatment was discontinued in 2 children treated with vancomycin, because of severe adverse events (renal impairment, 1 and neutropenia, 1), while all children in linezolid group were cured at the end of treatment. No differences were found between different cohorts in terms of adverse events, nausea and vomiting, diarrhea, skin rash, renal impairment, neutropenia, and thrombocytopenia. Patients in the linezolid group presented higher anemia rate [5 (83.3\%) vs $3(27.3 \%), \mathrm{P}=0.049]$ than those in the vancomycin group. None of peripheral neuropathy, visual impairment, RMS, nor hepatic impairment was observed in any of the patients.

\section{Discussion}

MRSA has become the most prevalent cause of AHO in pediatric patients[1]. Vancomycin therapy has been well established in children patients and demonstrates positive clinical outcomes. However, there are few options for children with renal impairment or intolerance to vancomycin. Linezolid could be an alternative to vancomycin for MRSA AHO[7], but it has been only approved for the use in pneumonia, bacteremia, skin infections, and periprosthetic joint infections caused by antibiotic-resistant Grampositive cocci[11, 22-24]. To date, no specific AHO study in children has been conducted[8, 10]. There is 
no literature comparing the clinical outcomes and adverse effects of linezolid and vancomycin for the treatment of specific MRSA AHO

As far as we know, this is the first research comparing the efficacy and safety of linezolid and vancomycin in the treatment of MRSA AHO. In aspects of clinical cure rates, microbiological eradiation, and hospital length of stay, linezolid and vancomycin have similar efficacy. No significantly differences were observed in most of the adverse events, apart from anemia.

It is difficult to compare our results with previous literature on MRSA AHO for the following reasons. First, the data provided by some previous studies cannot be compared, because the prescribed dose was unavailable or some other antibiotics were used with linezolid or vancomycin[4,5]. Second, previous studies on linezolid were limited by sparse AHO clinical outcome data coupled with concern over adverse effects with long term usage[9, 10,22,24]. To date no specific study of linezolid in the treatment of AHO has been conducted $[8,11,24]$. Thirdly, in other published articles with the treatment of linezolid or vancomycin in children, clinical outcomes were not compared between different antibiotics $[11,15,16$, 19].

Although no significant differences were observed in hemoglobin value at admission and EOT, the incidence rate of anemia was higher in the linezolid group $(5 / 6,83.3 \%)$ than in the vancomycin group $(3 / 11,27.3 \%, P=0.049)$, and the minimum value of hemoglobin in the ward also differed between treatment groups $(P=0.009)$. These findings seem to be inconsistent with previous studies that showed the incidence of anemia of $7.1 \%-15.3 \%$ with the treatment of linezolid in children[8, 10]. However, the treatment for MRSA AHO requires weeks of therapy[1] [30 (IQR 22-37) days in our study], and previous adverse effect data in children were limited to those seen when pediatric patients received linezolid for short duration $(11.3 \pm 5.0$ days) $[9,15]$. What's more, the total incidence rate of anemia previously was low, but 2 patients with anemia received linezolid for 32 and 36 days respectively, which was longer than the others' [20 (IQR 15-28) days][8]. According to a previous study in adults, pretreatment hemoglobin value of $<10.5 \mathrm{~g} / \mathrm{L}$ was an independent risk factor in patients who received more than 4 weeks of linezolid treatment[25]. However, in 3 patients with anemia $(60 \%)$ in our study, hemoglobin values were higher than $10.5 \mathrm{~g} / \mathrm{L}$ at admission. The prolonged use of linezolid was associated with an increased frequency of anemia, and children seemed to be more sensitive to the transient bone marrow suppression effect of linezolid. Additionally, in children with $\mathrm{AHO}$, factors including surgical blood loss, lesion range, and nutritional status may affect hemoglobin level, hence further prospective randomized controlled trials are needed to certify this observation.

The most common sites of infection were femur (52.9\%) and tibia (29.4\%), which were consistent with previous studies [26]. Inflammatory markers, including leukocyte, ESR, and CRP were compared at admission and EOT between treatment groups. Both CRP and ESR were elevated at the time of admission $(17 / 17>4 \mathrm{mg} / \mathrm{mL}, 17 / 17>20 \mathrm{~mm} /$ first $\mathrm{h}$, respectively), and declined to normal within therapy $(15 / 17$ $<4 \mathrm{mg} / \mathrm{mL}, 9 / 17<20 \mathrm{~mm} /$ first $\mathrm{h}$, respectively). This observation was compatible with that in previous articles [26-29]. According to a previous prospective study, leukocyte was an inappropriate predictor of 
response because only $35 \%$ of children demonstrated leukocytosis (leukocyte $>12 \times 10^{9} / \mathrm{L}$ ) [30]. In our study, leukocytosis only occurred in $5 / 17(29.4 \%)$ of children at admission, that is consistent with previous study [30].

The clinical chemistry criteria for DILI we used was applicable to patients of all ages[17], and no DILI was reported in this study. However, there is no independent diagnostic criteria for DILI in children[18], and the increase of ALP in serum is more common in children in the period of vigorous bone growth and development. Therefore, further studies are needed to explore potential DILI induced by linezolid or vancomycin. RMS is a rare but serious adverse reaction during treatment with intravenous antibiotics [20]. Although not observed in patients in this study, it occurred in one of our patients diagnosed with subacute hematogenous osteomyelitis (data not shown), and it was supposedly associated with rapid infusion of the first dose of the drug [21].

Discontinuation rate was higher in the vancomycin group $(2 / 11,18.2 \%)$ than in the linezolid group $(0 / 6$, $0.0 \%)$. This difference was not significant and may be caused by the sample size. The two failed patients underwent severe adverse events (renal impairment, 1, neutropenia, 1). Renal impairment caused by vancomycin in children was reported in several studies[16, 19, 31-33], and neutropenia associated with linezolid happened in $5(7.57 \%)$ children in a previous study[11]. However, few articles reported vancomycin related neutropenia in the treatment of $\mathrm{AHO}$ in children. Fortunately, the neutrophil level of this child returned to normal level $\left(>1.5 \times 10^{9} / \mathrm{L}\right)$ at EOT.

Interestingly, although no racial differences were observed in treatment outcomes and adverse events, ethnic minority children seemed to have a longer time between symptom onset and admission $(P=0.102)$ and a lower rate previous antimicrobial use before admission $(P=0.053)$ than Han children. It was suggested in previous studies that in cases of MRSA, patients should receive empirical antibiotic treatment at an early stage[26, 34]. This manifests the importance and greatness of the health poverty alleviation project proposed and carried out by our government, the aim of which is to provide basic needs to those still disadvantaged and in poor health, including improving their accessibility to, and affordability of, health services[35].

Therefore, with appropriate monitoring for signs of hematologic and toxicity, linezolid could be an alternative to vancomycin for the treatment of MRSA AHO in children. Additionally, its use allows patients to switch to the oral route more quickly, reducing the incidence of venous complications[8, 36, 37].

There are several limitations in this study. Although this study was non-randomized observational research, the authors followed strict inclusion criteria to decrease the potential bias. Despite this study used 8 years of data from our institution, only 17 MRSA AHO cases were included. Due to the small sample size, some differences in clinical outcomes and adverse events between treatment groups may be shadowed. However, to our knowledge, this study is the first to compare the clinical efficacy and safety of linezolid and vancomycin in the treatment of MRSA AHO in children. 


\section{Conclusion}

Although linezolid was considered an reliable alternative to vancomycin and has similar efficacy in the treatment of MRSA AHO. Children seemed to be more sensitive to the transient bone marrow suppression effect of linezolid in the prolonged use for MRSA AHO. The incidence rate of anemia was higher during long-term use of linezolid in children.

\section{Abbreviations}

MRSA, Methicillin-resistant Staphylococcus aureus; AHO, acute hematogenous osteomyelitis; IDSA, Infectious Diseases Society of America; IV, intravenous; EOT, end of treatment; LOS, hospital length of stay; RMS, red man syndrome; ESR, erythrocyte sedimentation rate; CRP, C-reactive protein; MICs, Minimal inhibitory concentrations; CLSI, Clinical and Laboratory Standards Institute; DILI, drug-induced liver injury; ULN, upper limit of normal; ALT, alanine aminotransferase; ALP, alkaline phosphatase; SCr, serum creatinine; IQR, interquartile range.

\section{Declarations}

\subsection{Ethics approval and consent to participate}

This study was approved by the Ethics Committee of Xijing Hospital. Informed consent was obtained from all participants or, if participants are under 18, from a parent and/or legal guardian.

\subsection{Consent for publication}

Not applicable.

\subsection{Availability of data and materials}

The data that support the findings of this study are available from Xijing Hospital, Air Force Military Medical University, however, restrictions apply regarding the availability of these data, which were used under a license for this study. The data may be available from reasonable request and with permission from Xijing Hospital. Please contact the corresponding author: HLY, huangly@fmmu.edu.cn.

\subsection{Competing interests}

No competing interests exist in this study.

\subsection{Funding}

The project is supported in part by the National Natural Science Foundation of China [Grant number: 81171735], the Provincial Natural Science Foundation of Shaanxi [Grant number: 2017JC2-04] and Air Force Military Medical University. 


\subsection{Authors' contributions}

HLY, XHF, and LC contributed to the study design. YYB, LC, LZC, DWWL, and GZX contributed to the data analysis and drafted the manuscript. LG directed data collection and provided administrative support for the project. All authors contributed to interpretation of the data, commented on the manuscript, revised the manuscript, revised the manuscript, and approved the final version for publication.

\subsection{Acknowledgements}

We appreciate the attending pediatric orthopedists of the Department of orthopedics who took care of the patients throughout the years. We thank Dr. G Li for guidance on statistics and data processing. We are particularly thankful to Dr. LY Huang and Dr. HF Xu for invaluable advice, encouragement, and interest in our project.

\section{References}

1. DeRonde KJ, Girotto JE, Nicolau DP. Management of Pediatric Acute Hematogenous Osteomyelitis, Part II: A Focus on Methicillin-Resistant Staphylococcus aureus, Current and Emerging Therapies. Pharmacotherapy. 2018,38(10):1021-37.

2. Loughran AJ, Gaddy D, Beenken KE, Meeker DG, Morello R, Zhao H, et al. Impact of sarA and PhenolSoluble Modulins on the Pathogenesis of Osteomyelitis in Diverse Clinical Isolates of Staphylococcus aureus. Infect Immun. 2016,84(9):2586-94.

3. Martínez-Aguilar G, Avalos-Mishaan A, Hulten K, Hammerman W, Mason EO Jr, Kaplan SL. Community-acquired, methicillin-resistant and methicillin-susceptible Staphylococcus aureus musculoskeletal infections in children. Pediatr Infect Dis J. 2004,23(8):701-6.

4. Arnold SR, Elias D, Buckingham SC, Thomas ED, Novais E, Arkader A, et al. Changing patterns of acute hematogenous osteomyelitis and septic arthritis: emergence of community-associated methicillin-resistant Staphylococcus aureus. J Pediatr Orthop. 2006,26(6):703-8.

5. McNeil JC, Kaplan SL, Vallejo JG. The Influence of the Route of Antibiotic Administration, Methicillin Susceptibility, Vancomycin Duration and Serum Trough Concentration on Outcomes of Pediatric Staphylococcus aureus Bacteremic Osteoarticular Infection. Pediatr Infect Dis J. 2017,36(6):572-7.

6. Kollef MH. Limitations of vancomycin in the management of resistant staphylococcal infections. Clin Infect Dis. 2007,45 Suppl 3:S191-5.

7. Liu C, Bayer A, Cosgrove SE, Daum RS, Fridkin SK, Gorwitz RJ, et al. Clinical practice guidelines by the infectious diseases society of america for the treatment of methicillin-resistant Staphylococcus aureus infections in adults and children. Clin Infect Dis. 2011,52(3):e18-55.

8. Chen CJ, Chiu CH, Lin TY, Lee ZL, Yang WE, Huang YC. Experience with linezolid therapy in children with osteoarticular infections. Pediatr Infect Dis J. 2007,26(11):985-8.

9. Cojutti P, Maximova N, Crichiutti G, Isola M, Pea F. Pharmacokinetic/pharmacodynamic evaluation of linezolid in hospitalized paediatric patients: a step toward dose optimization by means of 
therapeutic drug monitoring and Monte Carlo simulation. J Antimicrob Chemother. 2015,70(1):198206.

10. Kaplan SL, Deville JG, Yogev R, Morfin MR, Wu E, Adler S, et al. Linezolid versus vancomycin for treatment of resistant Gram-positive infections in children. Pediatr Infect Dis J. 2003,22(8):677-86.

11. Kaplan SL, Patterson L, Edwards KM, Azimi PH, Bradley JS, Blumer JL, et al. Linezolid for the treatment of community-acquired pneumonia in hospitalized children. Linezolid Pediatric Pneumonia Study Group. Pediatr Infect Dis J. 2001,20(5):488-94.

12. Flamm RK, Mendes RE, Hogan PA, Streit JM, Ross JE, Jones RN. Linezolid Surveillance Results for the United States (LEADER Surveillance Program 2014). Antimicrob Agents Chemother. 2016,60(4):2273-80.

13. Ma B, Niu C, Zhou Y, Xue X, Meng J, Luo X, et al. The Disulfide Bond of the Peptide Thanatin Is Dispensible for Its Antimicrobial Activity In Vivo and In Vitro. Antimicrob Agents Chemother. 2016,60(7):4283-9.

14. Corey GR, Good S, Jiang H, Moeck G, Wikler M, Green S, et al. Single-dose oritavancin versus 7-10 days of vancomycin in the treatment of gram-positive acute bacterial skin and skin structure infections: the SOLO II noninferiority study. Clin Infect Dis. 2015,60(2):254-62.

15. Nambiar S, Rellosa N, Wassel RT, Borders-Hemphill V, Bradley JS. Linezolid-associated peripheral and optic neuropathy in children. Pediatrics. 2011,127(6):e1528-32.

16. Sinclair EA, Yenokyan G, McMunn A, Fadrowski JJ, Milstone AM, Lee CK. Factors associated with acute kidney injury in children receiving vancomycin. Ann Pharmacother. 2014,48(12):1555-62.

17. Aithal GP, Watkins PB, Andrade RJ, Larrey D, Molokhia M, Takikawa $H$, et al. Case definition and phenotype standardization in drug-induced liver injury. Clin Pharmacol Ther. 2011,89(6):806-15.

18. Björnsson ES. Drug-induced liver injury: an overview over the most critical compounds. Arch Toxicol. 2015,89(3):327-34.

19. McKamy S, Hernandez E, Jahng M, Moriwaki T, Deveikis A, Le J. Incidence and risk factors influencing the development of vancomycin nephrotoxicity in children. J Pediatr. 2011,158(3):422-6.

20. Bauters T, Claus B, Schelstraete P, Robays H, Benoit Y, Dhooge C. Vancomycin-induced red man syndrome in pediatric oncology: still an issue. Int J Clin Pharm. 2012,34(1):13-6.

21. Sivagnanam S, Deleu D. Red man syndrome. Crit Care. 2003,7(2):119-20.

22. Sawada M, Oe K, Hirata M, Kawamura H, Ueda N, Nakamura T, et al. Linezolid versus daptomycin treatment for periprosthetic joint infections: a retrospective cohort study. J Orthop Surg Res. 2019,14(1):334.

23. Tan TQ. Update on the use of linezolid: a pediatric perspective. Pediatr Infect Dis J. 2004,23(10):9556.

24. Wible K, Tregnaghi M, Bruss J, Fleishaker D, Naberhuis-Stehouwer S, Hilty M. Linezolid versus cefadroxil in the treatment of skin and skin structure infections in children. Pediatr Infect Dis J. 2003,22(4):315-23. 
25. Senneville E, Legout L, Valette M, Yazdanpanah Y, Giraud F, Beltrand E, et al. Risk factors for anaemia in patients on prolonged linezolid therapy for chronic osteomyelitis: a case-control study. J Antimicrob Chemother. 2004,54(4):798-802.

26. Dartnell J, Ramachandran M, Katchburian M. Haematogenous acute and subacute paediatric osteomyelitis: a systematic review of the literature. J Bone Joint Surg Br. 2012,94(5):584-95.

27. DeRonde KJ, Girotto JE, Nicolau DP. Management of Pediatric Acute Hematogenous Osteomyelitis, Part I: Antimicrobial Stewardship Approach and Review of Therapies for Methicillin-Susceptible Staphylococcus aureus, Streptococcus pyogenes, and Kingella kingae. Pharmacotherapy. 2018,38(9):947-66.

28. Peltola H, Unkila-Kallio L, Kallio MJ. Simplified treatment of acute staphylococcal osteomyelitis of childhood. The Finnish Study Group. Pediatrics. 1997,99(6):846-50.

29. Saavedra-Lozano J, Mejías A, Ahmad N, Peromingo E, Ardura MI, Guillen S, et al. Changing trends in acute osteomyelitis in children: impact of methicillin-resistant Staphylococcus aureus infections. J Pediatr Orthop. 2008,28(5):569-75.

30. Unkila-Kallio L, Kallio MJ, Eskola J, Peltola H. Serum C-reactive protein, erythrocyte sedimentation rate, and white blood cell count in acute hematogenous osteomyelitis of children. Pediatrics. 1994,93(1):59-62.

31. Cies JJ, Shankar V. Nephrotoxicity in patients with vancomycin trough concentrations of $15-20$ $\mu \mathrm{g} / \mathrm{ml}$ in a pediatric intensive care unit. Pharmacotherapy. 2013,33(4):392-400.

32. Faden $D$, Faden HS. The high rate of adverse drug events in children receiving prolonged outpatient parenteral antibiotic therapy for osteomyelitis. Pediatr Infect Dis J. 2009,28(6):539-41.

33. Gomez M, Maraqa N, Alvarez A, Rathore M. Complications of outpatient parenteral antibiotic therapy in childhood. Pediatr Infect Dis J. 2001,20(5):541-3.

34. Gwynne-Jones DP, Stott NS. Community-acquired methicillin-resistant Staphylococcus aureus: a cause of musculoskeletal sepsis in children. J Pediatr Orthop. 1999,19(3):413-6.

35. Wang YP, Zhou XN. The year 2020, a milestone in breaking the vicious cycle of poverty and illness in China. Infect Dis Poverty. 2020,9(1):11.

36. Echeverria-Esnal D, Sorli L, Prim N, Conde-Estévez D, Mateu-De Antonio J, Martin-Ontiyuelo C, et al. Linezolid vs glycopeptides in the treatment of glycopeptide-susceptible Enterococcus faecium bacteraemia: A propensity score matched comparative study. Int J Antimicrob Agents. 2019,54(5):572-8.

37. Mouwen A, Dijkstra JA, Jong E, Buijtels P, Pasker-de Jong P, Nagtegaal JE. Early switching of antibiotic therapy from intravenous to oral using a combination of education, pocket-sized cards and switch advice: A practical intervention resulting in reduced length of hospital stay. Int $\mathrm{J}$ Antimicrob Agents. 2020,55(1):105769.

\section{Tables}


Due to technical limitations, tables are only available as a download in the Supplemental Files section.

\section{Supplementary Files}

This is a list of supplementary files associated with this preprint. Click to download.

- TABLES.docx 